\title{
Nontrivial Solutions for a Modified Capillary Surface Equation
}

\author{
Zhanping Liang \\ School of Mathematical Sciences, Shanxi University, Taiyuan, Shanxi 030006, China \\ Correspondence should be addressed to Zhanping Liang; lzp@sxu.edu.cn
}

Received 1 December 2012; Accepted 6 February 2013

Academic Editor: James H. Liu

Copyright (C) 2013 Zhanping Liang. This is an open access article distributed under the Creative Commons Attribution License, which permits unrestricted use, distribution, and reproduction in any medium, provided the original work is properly cited.

A negative solution and a positive solution are obtained for a modified capillary surface equation by variational methods.

\section{Introduction}

In this paper, we study the existence of nontrivial solutions to the following quasilinear elliptic equation:

$$
\begin{gathered}
-\operatorname{div}\left(\frac{|\nabla u|^{2 p-2} \nabla u}{\sqrt{1+|\nabla u|^{2 p}}}\right)=f(x, u) \quad \text { in } \Omega, \\
u=0 \quad \text { on } \partial \Omega,
\end{gathered}
$$

where $p>1$, and $\Omega$ is a bounded domain in $\mathbb{R}^{N}$ with smooth boundary. The function $f \in C(\bar{\Omega} \times \mathbb{R}, \mathbb{R})$ with the subcritical growth

(f)

$$
|f(x, t)| \leq c\left(1+|t|^{q-1}\right), \quad t \in \mathbb{R}, x \in \Omega,
$$

where $q \in[1, N p /(N-p))$ if $1<p<N$ or $q \in[1,+\infty)$ if $1<N \leq p$, and $c$ is a positive constant.

In the case that $p=1,(1)$ is the mean curvature equation or the capillary surface equation; when $f(x, u) \equiv u$, it describes the equilibrium shape of a liquid surface with constant surface tension in a uniform gravity field, and this is the shape of a pendent drop [1]. When $p>1$, one calls (1) a modified capillary surface equation which is also worth considering even though it is not exactly the capillary surface equation [2]. For the capillary surface equation, radially symmetric solutions in the case that $\Omega$ is a ball or entire space have been investigated precisely; See, for example, [3-5] and the references therein. In [2], by minimization sequence method and the Ambrosetti-Rabinowitz mountain pass lemma without Palais-Smale condition, positive solutions were obtained to nonlinear eigenvalue problem for the modified capillary surface equation which is of the form

$$
\begin{gathered}
-\operatorname{div}\left(\frac{|\nabla u|^{2 p-2} \nabla u}{\sqrt{1+|\nabla u|^{2 p}}}\right)=\lambda f(x, u) \quad \text { in } \Omega, \\
u \geq 0 \quad \text { in } \Omega, \\
u=0 \quad \text { on } \partial \Omega,
\end{gathered}
$$

where $\lambda$ is a positive parameter. In the proof of the main results of [2], $\lambda$ is crucial not only to the existence of global or local minimizer but also to the construction of mountain pass geometry. In our paper, one object is to find existence conditions of solutions to (1) without the constraint of $\lambda$. Since

$$
\sqrt{1+|\nabla u|^{2 p}}-1 \sim|\nabla u|^{p} \text { as }|\nabla u| \longrightarrow \infty,
$$

the other object is to investigate the probability to present the property of $f$ by the eigenvalue of the problem

$$
\begin{gathered}
-\Delta_{p} u=\lambda|u|^{p-2} u \quad \text { in } \Omega, \\
u=0 \quad \text { on } \partial \Omega,
\end{gathered}
$$

where $\Delta_{p} u=\operatorname{div}\left(|\nabla u|^{p-2} \nabla u\right)$. 
In the following, we recall some known facts about problem (5). Let $\lambda_{1}>0$ be the first eigenvalue of the problem (5). It is known that $\lambda_{1}$ is characterized by

$$
\lambda_{1}:=\inf \left\{\int_{\Omega}|\nabla u|^{p} d x: \int_{\Omega}|u|^{p} d x=1, u \in W_{0}^{1, p}(\Omega) \backslash\{0\}\right\},
$$

where $W_{0}^{1, p}(\Omega)$ is the reflexive Banach space defined as the completion of $C_{0}^{\infty}(\Omega)$ with respect to the norm $\|u\|:=$ $\left(\int_{\Omega}|\nabla u|^{p} d x\right)^{1 / p}$. Also, $\lambda_{1}$ is single and has an associated eigenfunction $\varphi_{1}>0$ in $\Omega$ and $\left\|\varphi_{1}\right\|=1$. The reader is referred to $[6,7]$ for details.

By a solution $u$ of (1), we mean that $u$ satisfies (1) in the weak sense; that is, for all $\varphi \in W_{0}^{1, p}(\Omega)$,

$$
\int_{\Omega} \frac{|\nabla u|^{2 p-2} \nabla u \nabla \varphi}{\sqrt{1+|\nabla u|^{2 p}}} d x=\int_{\Omega} f(x, u) \varphi d x
$$

A solution such that $u(x) \geq 0$ in $\Omega$ and $u \neq 0$, respectively, $u(x) \leq 0$ in $\Omega$ and $u \neq 0$, is a positive, respectively, negative, solution.

Define

$$
\begin{gathered}
J(u)=\frac{1}{p} \int_{\Omega}\left(\sqrt{1+|\nabla u|^{2 p}}-1\right) d x, \quad u \in W_{0}^{1, p}(\Omega), \\
K(u)=\int_{\Omega} F(x, u) d x, \quad u \in W_{0}^{1, p}(\Omega), \\
I(u)=J(u)-K(u), \quad u \in W_{0}^{1, p}(\Omega),
\end{gathered}
$$

where $F(x, t)=\int_{0}^{t} f(x, s) d s$. From a variational stand point, finding solutions of $(1)$ in $W_{0}^{1, p}(\Omega)$ is equivalent to finding critical points of the $C^{1}$ functional $I$. As to the differentiability of the functional $I$, one can consult [2] for details. Since $f$ satisfies the subcritical growth condition $\left(f_{0}\right)$, stand proofs show that $K$ is weakly continuous. Since the function $\varphi(t)=$ $\sqrt{1+t^{2 p}}$ is convex, the functional $J$ is also convex. In addition, $J$ belongs to $C^{1}$. Hence, $J$ is weakly lower semicontinuous. Thus, we have shown that $I$ is weakly lower semicontinuous.

Now, let us state the main results of this paper.

Theorem 1. Let $(f)$ hold. Furthermore, assume that $f$ satisfies the following conditions.

$\left(f_{0}\right)$ There is some $r>0$ small such that

$$
p F(x, t) \geq \lambda_{1}|t|^{p}, \quad|t| \leq r, x \in \Omega
$$

$\left(f_{1}\right) \lim \sup _{|t| \rightarrow \infty}\left(p F(x, t) /|t|^{p}\right)<\lambda_{1}$ uniformly for $x \in \Omega$.

Then, (1) has at least a negative solution and a positive solution which correspond to negative critical values of the associated functional given by (8).
Theorem 2. Let $(f)$ and $\left(f_{0}\right)$ hold. Furthermore, assume that $f$ satisfies the following conditions.

$\left(f_{2}\right) \lim _{|t| \rightarrow \infty}\left(p F(x, t) /|t|^{p}\right)=\lambda_{1}$ uniformly for $x \in$ $\Omega$,

$\left(f_{3}\right) \lim _{|t| \rightarrow \infty}(f(x, t)-p F(x, t))=+\infty$ uniformly for $x \in \Omega$.

Then, (1) has at least a negative solution and a positive solution which correspond to negative critical values of the associated functional given by (8).

Remark 3. With the conditions $\left(f_{0}\right)-\left(f_{3}\right)$, Liu and $\mathrm{Su}$ in [8] have studied the existence of solutions to $p$-Laplacian quasilinear elliptic equation

$$
\begin{gathered}
-\Delta_{p} u=f(x, u) \quad \text { in } \Omega, \\
u=0 \quad \text { on } \partial \Omega .
\end{gathered}
$$

Under the conditions $\left(f_{0}\right)$ and $\left(f_{1}\right),(10)$ may be resonant at the eigenvalue $\lambda_{1}$ near the origin. With the conditions $\left(f_{2}\right)$ and $\left(f_{3}\right)$, it may be resonant at $\lambda_{1}$ both near the origin and near infinity. In fact, the condition $\left(f_{0}\right)$ allows $(10)$ to be resonant near the origin from the right side of $\lambda_{1}$, while the conditions $\left(f_{2}\right)$ and $\left(f_{3}\right)$ allow it to be resonant at infinity from the left side of $\lambda_{1}$.

Remark 4. Theorems 1 and 2 have shown a new fact that the interaction between the first eigenvalue of $-\Delta_{p}$ with zero Dirichlet boundary data and nonlinearity $f$ can influence the existence of nontrivial solutions to (1).

Before concluding this section, we explain some notations used in the paper. $|\Omega|$ is the Lebesgue measure of $\Omega$. $c_{i}(i \in \mathbb{N})$ is always a positive constant independent of functions. $\langle\cdot, \cdot\rangle$ is the duality between $\left(W_{0}^{1, p}(\Omega)\right)^{*}$ and $W_{0}^{1, p}(\Omega)$. In addition, we use $|\cdot|$ to denote the usual norm of $\mathbb{R}^{N}$.

\section{The Proof of the Main Results}

In this section, we prove Theorems 1 and 2.

Proof of Theorem 1. The proof consists of two steps.

(i) To obtain a positive solution, cut-off techniques are used. Define

$$
\begin{gathered}
\widehat{f}(x, t)= \begin{cases}f(x, t), & t \geq 0, \\
0, & t<0,\end{cases} \\
\widehat{F}(x, t)=\int_{0}^{t} \widehat{f}(x, s) d s, \\
\widehat{I}(u)=J(u)-\int_{\Omega} \widehat{F}(x, u) d x, \quad u \in W_{0}^{1, p}(\Omega) .
\end{gathered}
$$

Since $f \in C(\bar{\Omega} \times \mathbb{R}, \mathbb{R})$ and $\left(f_{1}\right)$ holds, for any given $\varepsilon>0$, there exists $c_{1}>0$ such that

$$
\widehat{F}(x, t) \leq \frac{1}{p}\left(\lambda_{1}-\varepsilon\right)|t|^{p}+c_{1}, \quad t \in \mathbb{R}, x \in \Omega .
$$


By the Poincaré inequality, for $u \in W_{0}^{1, p}(\Omega)$,

$$
\begin{aligned}
\widehat{I}(u)= & \frac{1}{p} \int_{\Omega} \sqrt{1+|\nabla u|^{2 p}} d x-\int_{\Omega} \widehat{F}(x, u) d x-\frac{|\Omega|}{p} \\
\geq & \frac{1}{p} \int_{\Omega}|\nabla u|^{p} d x-\frac{1}{p}\left(\lambda_{1}-\varepsilon\right) \int_{\Omega}|u|^{p} d x \\
& -\left(c_{1}+\frac{1}{p}\right)|\Omega| \\
\geq & \frac{1}{p}\left(1-\frac{\lambda_{1}-\varepsilon}{\lambda_{1}}\right)\|u\|^{p}-\left(c_{1}+\frac{1}{p}\right)|\Omega| \\
= & \frac{\varepsilon}{p \lambda_{1}}\|u\|^{p}-\left(c_{1}+\frac{1}{p}\right)|\Omega| .
\end{aligned}
$$

Hence, $\widehat{I}$ is coercive; that is, $\widehat{I}(u) \rightarrow \infty$ as $n \rightarrow \infty$. In addition, since $\widehat{f}$ also satisfies the condition $(f), \widehat{I}$ is weakly lower semi-continuous. So, it has a global minimizer.

Take a number $t_{0}>0$ such that $0<t_{0} \varphi_{1} \leq r$ in $\Omega$. By the condition $\left(f_{0}\right)$, we have that

$$
\begin{aligned}
\widehat{I}\left(t_{0} \varphi_{1}\right)= & \frac{1}{p} \int_{\Omega}\left(\sqrt{1+t_{0}^{2 p}\left|\nabla \varphi_{1}\right|^{2 p}}-1\right) d x \\
& -\int_{\Omega} F\left(x, t_{0} \varphi_{1}\right) d x \\
< & \frac{1}{p} t_{0}^{p} \int_{\Omega}\left|\nabla \varphi_{1}\right|^{p} d x-\frac{1}{p} \lambda_{1} t_{0}^{p} \int_{\Omega} \varphi_{1}^{p} d x \\
= & 0 .
\end{aligned}
$$

Thus, the global minimizer of $\widehat{I}$ is a nontrivial critical point, denoted by $u_{1}$ which satisfies $\widehat{I}\left(u_{1}\right)<0$. Putting $u_{1}^{-}(x)=$ $\min \left\{u_{1}(x), 0\right\}$, we have that

$$
\left\langle\widehat{I}^{\prime}\left(u_{1}\right), u_{1}^{-}\right\rangle=\int_{\Omega} \frac{\left|\nabla u_{1}^{-}\right|^{2 p}}{\sqrt{1+\left|\nabla u_{1}^{-}\right|^{2 p}}} d x=0 .
$$

Hence, $u_{1}^{-}=0$. So, $u_{1}$ is a positive solution of $(1)$, and $I\left(u_{1}\right)<$ 0 . $\hat{f}$ with

(ii) To obtain a negative solution, we only need to replace

$$
\tilde{f}(x, t)= \begin{cases}0, & t>0, \\ f(x, t), & t \leq 0 .\end{cases}
$$

Similar to step (i), it is shown that (1) has a negative solution $u_{2}$ with $I\left(u_{2}\right)<0$.

The proof is completed.

Proof of Theorem 2. We adopt the notations in the proof of Theorem 1.

First of all, we show that the functional $\widehat{I}$ is also coercive under the conditions $\left(f_{2}\right)$ and $\left(f_{3}\right)$. Write

$$
\begin{aligned}
& \widehat{F}(x, t)=\frac{1}{p} \lambda_{1}\left(t^{+}\right)^{p}+\widehat{G}(x, t), \\
& \widehat{f}(x, t)=\lambda_{1}|t|^{p-2} t^{+}+\widehat{g}(x, t),
\end{aligned}
$$

where $t^{+}=\max \{t, 0\}$. Given $x \in \Omega$, we have that

$$
\begin{gathered}
\lim _{t \rightarrow+\infty} \frac{p \widehat{G}(x, t)}{t^{p}}=0, \\
\lim _{t \rightarrow+\infty}(\widehat{g}(x, t) t-p \widehat{G}(x, t))=+\infty .
\end{gathered}
$$

Thus, for every $M>0$, there exists $R_{M}>0$ such that

$$
\widehat{g}(x, t) t-p \widehat{G}(x, t) \geq M, \quad t \geq R_{M}, x \in \Omega .
$$

Integrating the equality

$$
\frac{d}{d t}\left(\frac{\widehat{G}(x, t)}{t^{p}}\right)=\frac{\widehat{g}(x, t) t-p \widehat{G}(x, t)}{t^{p+1}}
$$

over the interval $[t, T] \subset\left[R_{M},+\infty\right)$,

$$
\frac{\widehat{G}(x, T)}{T^{p}}-\frac{\widehat{G}(x, t)}{t^{p}} \geq \frac{M}{p}\left(\frac{1}{t^{p}}-\frac{1}{T^{p}}\right) .
$$

Letting $T \rightarrow+\infty$, we show that $\widehat{G}(x, t) \leq-M / p, t \geq R_{M}$.

Suppose that $\left\{u_{n}\right\} \subset W_{0}^{1, p}(\Omega)$ satisfies $\left\|u_{n}\right\| \rightarrow \infty$ and $\widehat{I}\left(u_{n}\right) \leq C$ for some constant $C \in \mathbb{R}$. Let $v_{n}=u_{n} /\left\|u_{n}\right\|$. Up to subsequence if necessary, we may assume that there exists $v_{0} \in W_{0}^{1, p}(\Omega)$ such that

$$
\begin{gathered}
v_{n} \rightarrow v_{0} \quad \text { in } E, \\
v_{n} \longrightarrow v_{0} \quad \text { in } L^{p}(\Omega), \\
v_{n}(x) \longrightarrow v_{0}(x) \quad \text { a.e. } x \in \Omega .
\end{gathered}
$$

Given $M=1$ in (19), we have that

$$
\widehat{G}(x, t) \leq-\frac{1}{p}, \quad t \geq R_{1} .
$$

Let $c_{2}=\max _{(x, t) \in \bar{\Omega} \times\left[-R_{1}, R_{1}\right]}|\widehat{G}(x, t)|$. Thus,

$$
\begin{aligned}
\frac{C}{\left\|u_{n}\right\|^{p} \geq} & \frac{1}{p\left\|u_{n}\right\|^{p}}\left(\int_{\Omega} \sqrt{1+|\nabla u|^{2 p}} d x-\lambda_{1} \int_{\Omega}\left|u_{n}\right|^{p} d x\right) \\
& -\frac{1}{\left\|u_{n}\right\|^{p}} \int_{\Omega} \widehat{G}\left(x, u_{n}\right) d x-\frac{|\Omega|}{p\left\|u_{n}\right\|^{p}} \\
\geq & \frac{1}{p} \int_{\Omega}\left(\left|\nabla v_{n}\right|^{p}-\lambda_{1}\left|v_{n}\right|^{p}\right) d x-\frac{|\Omega|}{p\left\|u_{n}\right\|^{p}} \\
& -\frac{1}{\left\|u_{n}\right\|^{p}} \int_{\left|u_{n}\right| \geq R_{1}} \widehat{G}\left(x, u_{n}\right) d x \\
& -\frac{1}{\left\|u_{n}\right\|^{p}} \int_{\left|u_{n}\right| \leq R_{1}} \widehat{G}\left(x, u_{n}\right) d x \\
\geq & \frac{1}{p} \int_{\Omega}\left(\left|\nabla v_{n}\right|^{p}-\lambda_{1}\left|v_{n}\right|^{p}\right) d x-\frac{|\Omega|}{p\left\|u_{n}\right\|^{p}}-\frac{c_{2}|\Omega|}{\left\|u_{n}\right\|^{p}} \\
= & \frac{1}{p} \int_{\Omega}\left(\left|\nabla v_{n}\right|^{p}-\lambda_{1}\left|v_{n}\right|^{p}\right) d x+\frac{c_{3}}{\left\|u_{n}\right\|^{p}},
\end{aligned}
$$


where $c_{3}=\left(1 / p+c_{2}\right)|\Omega|$. It follows from (22) and the previous inequality that

$$
\limsup _{n \rightarrow \infty} \int_{\Omega}\left|\nabla v_{n}\right|^{p} d x \leq \lambda_{1} \int_{\Omega}\left|v_{0}\right|^{p} d x .
$$

Because the norm is weakly lower semi-continuous, using Poincaré inequality, we get that

$$
\begin{aligned}
\limsup _{n \rightarrow \infty} \int_{\Omega}\left|\nabla v_{n}\right|^{p} d x & \leq \lambda_{1} \int_{\Omega}\left|v_{0}\right|^{p} d x \leq \int_{\Omega}\left|\nabla v_{0}\right|^{p} d x \\
& \leq \liminf _{n \rightarrow \infty} \int_{\Omega}\left|\nabla v_{n}\right|^{p} d x \\
& \leq \limsup _{n \rightarrow \infty} \int_{\Omega}\left|\nabla v_{n}\right|^{p} d x .
\end{aligned}
$$

Hence, $\int_{\Omega}\left|\nabla v_{0}\right|^{p} d x=\lambda_{1} \int_{\Omega}\left|v_{0}\right|^{p} d x$ and $v_{n} \rightarrow v_{0}$ in $W_{0}^{1, p}(\Omega)$ with $\left\|v_{0}\right\|=1$. So, $v_{0}$ is the corresponding eigenfunction to $\lambda_{1}$. Without loss of generality, we may assume that $v_{0}=\varphi_{1}$. Thus, $u_{n} \rightarrow+\infty$ a.e. $x \in \Omega$. Consequently, $\widehat{G}\left(x, u_{n}(x)\right) \rightarrow-\infty$ a.e. $x \in \Omega$. Therefore,

$$
C \geq-\int_{\Omega} \widehat{G}\left(x, u_{n}\right) d x \longrightarrow+\infty,
$$

which contradicts the fact that $C \in \mathbb{R}$. From the fact that $\widehat{I}$ is weakly low semi-continuous, we know that it has a global minimizer $u_{1}$. As in the proof of Theorem $1, u_{1}$ is a positive solution of $(1)$ with $I\left(u_{1}\right)<0$. In a similar way, we can obtain a negative solution with negative critical value.

The proof is completed.

\section{Acknowledgments}

The author would like to express sincere thanks to the anonymous referee whose careful reading and valuable comments improved the paper. This work is partially supported by the National Natural Science Foundation of China (Grant no. 11071149) and Science Council of Shanxi Province (2010011001-1 and 2012011004-2).

\section{References}

[1] R. Finn, Equilibrium Capillary Surfaces, Springer, New York, NY, USA, 1986.

[2] K. Narukawa and T. Suzuki, "Nonlinear eigenvalue problem for a modified capillary surface equation," Funkcialaj Ekvacioj, vol. 37, no. 1, pp. 81-100, 1994.

[3] P. Concus and R. Finn, "The shape of a pendent liquid drop," Philosophical Transactions of the Royal Society of London A, vol. 292, no. 1391, pp. 307-340, 1978.

[4] K. Narukawa and T. Suzuki, "Nonlinear eigenvalue problem associated to capillary surfaces," Unione Matematica Italiana. Bollettino. B. Serie VII, vol. 4, no. 1, pp. 223-241, 1990.

[5] H. C. Wente, "The symmetry of sessile and pendent drops," Pacific Journal of Mathematics, vol. 88, no. 2, pp. 387-397, 1980.

[6] A. Anane, "Simplicité et isolation de la première valeur propre du p-laplacien avec poids," Comptes Rendus des Séances de l'Académie des Sciences, vol. 305, no. 16, pp. 725-728, 1987.
[7] P. Lindqvist, "On the equation $\left(|\nabla u|^{p-2} \nabla u\right)+\lambda|u|^{p-2} u=0$," Proceedings of the American Mathematical Society, vol. 109, no. 1, pp. 157-164, 1990.

[8] J. Liu and J. Su, "Remarks on multiple nontrivial solutions for quasi-linear resonant problems," Journal of Mathematical Analysis and Applications, vol. 258, no. 1, pp. 209-222, 2001. 


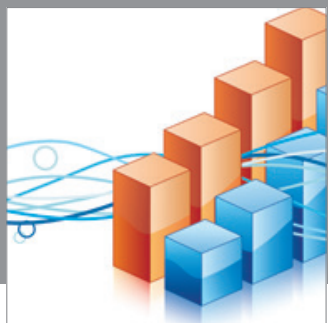

Advances in

Operations Research

mansans

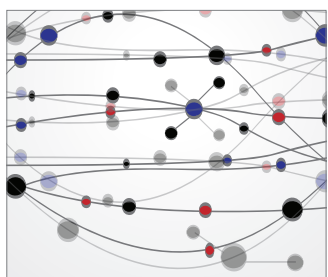

The Scientific World Journal
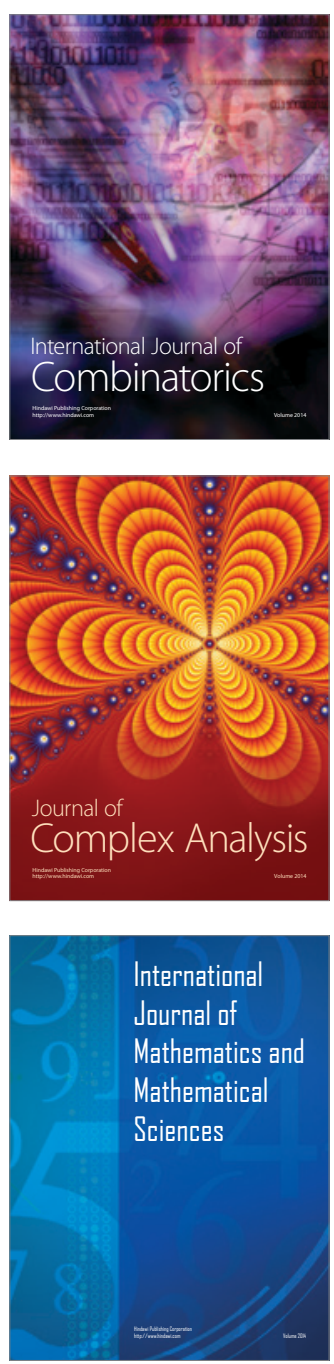
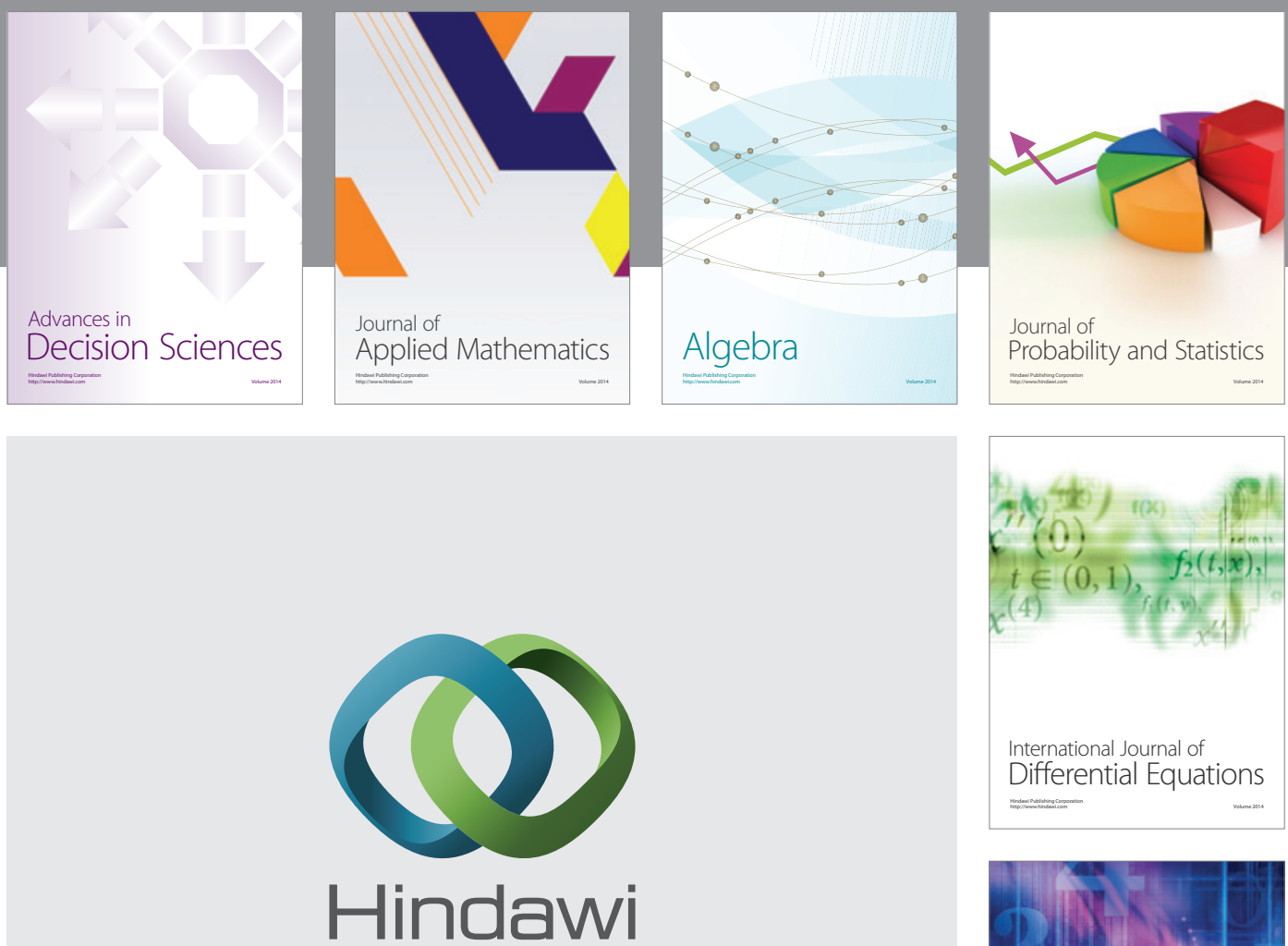

Submit your manuscripts at http://www.hindawi.com
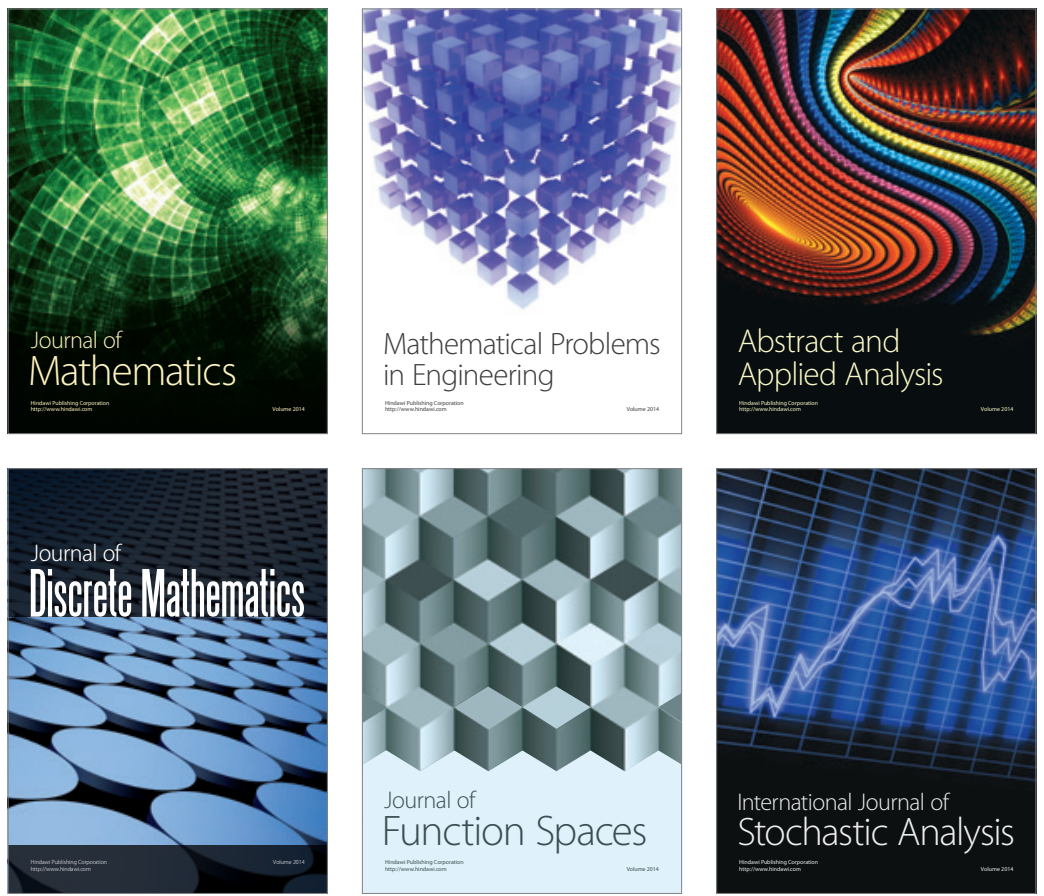

Journal of

Function Spaces

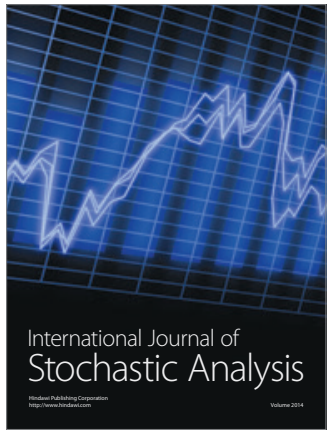

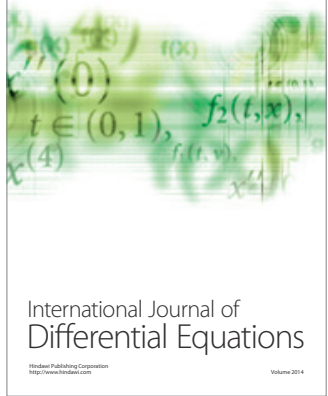
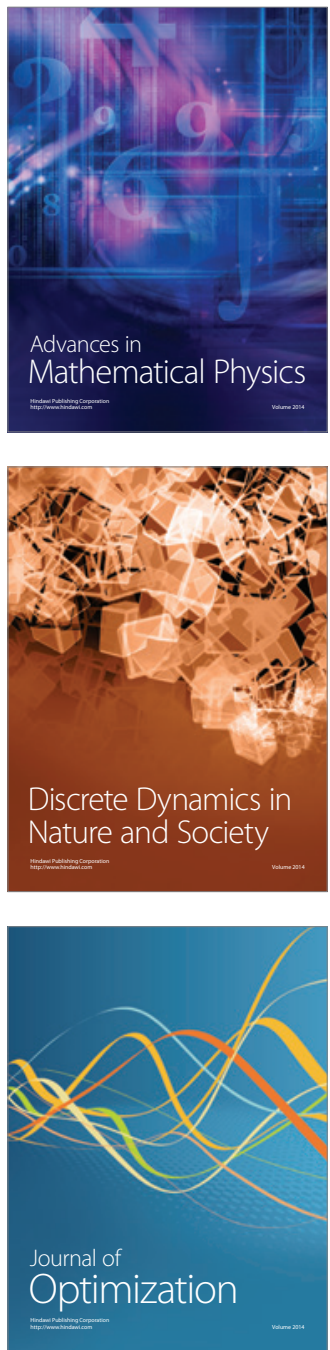